\title{
Old World cutaneous leishmaniasis treatment response varies depending on parasite species, geographical location and development of secondary infection
}

\author{
Waleed S. Al-Salem ${ }^{1,2 \dagger}{ }^{1}$, Carla Solórzano ${ }^{3 \dagger}$, Gareth D. Weedall4 ${ }^{4}$ Naomi A. Dyer ${ }^{1}$, Louise Kelly-Hope ${ }^{1}$, \\ Aitor Casas-Sánchez ${ }^{1}$, Yasser Alraey ${ }^{1}$, Essam J. Alyamani ${ }^{5}$, Alice Halliday ${ }^{6}$, Salah M. Balghonaim7, \\ Khalid S. Alsohibany ${ }^{7}$, Zeyad Alzeyadi ${ }^{8}$, Mohamed H. Alzahrani ${ }^{7}$, Ali M. Al-Shahrani ${ }^{7}$, Abdullah M. Assiri ${ }^{7}$, \\ Ziad Memish ${ }^{7}$ and Álvaro Acosta-Serrano ${ }^{1,9^{*}}$ (D)
}

\begin{abstract}
Background: In the Kingdom of Saudi Arabia (KSA), Leishmania major and L. tropica are the main causative agents of Old World cutaneous leishmaniasis (CL). The national $\mathrm{CL}$ treatment regimen consists of topical 1\% clotrimazole/2\% fusidic acid cream followed by $1-2$ courses of intralesional sodium stibogluconate (SSG); however, treatment efficacy is highly variable and the reasons for this are not well understood. In this study, we present a complete epidemiological map of $C L$ and determined the efficacy of the standard $C L$ treatment regime in several endemic regions of KSA.

Results: Overall, three quarters of patients in all CL-endemic areas studied responded satisfactorily to the current treatment regime, with the remaining requiring only an extra course of SSG. The majority of unresponsive cases were infected with L. tropica. Furthermore, the development of secondary infections (SI) around or within the CL lesion significantly favoured the treatment response of $L$. major patients but had no effect on $L$. tropica cases.

Conclusions: The response of $C L$ patients to a national treatment protocol appears to depend on several factors, including Leishmania parasite species, geographical location and occurrences of SI. Our findings suggest there is a need to implement alternative $C L$ treatment protocols based on these parameters.
\end{abstract}

Keywords: Cutaneous leishmaniasis, Saudi Arabia, Epidemiology, Treatment response, Secondary infections

\section{Background}

Old World cutaneous leishmaniasis (CL) is endemic throughout the East Mediterranean Region (EMR) [1]. According to the World Health Organization, in recent years, over 150,000 human CL cases were reported in 16 EMR countries [2]. Various factors contribute to the spread of $\mathrm{CL}$ in this region, including uncontrolled urbanization, irrigation, governmental sector integration,

\footnotetext{
*Correspondence: alvaro.acosta-serrano@lstmed.ac.uk

tWaleed S. Al-Salem and Carla Solórzano contributed equally to this work ${ }^{1}$ Department of Tropical Disease Biology, Liverpool School of Tropical Medicine, Liverpool, UK

Full list of author information is available at the end of the article
}

socio-economic factors and lack of health education [3] War is another important factor responsible for CL outbreaks in the region, with over 200,000 people infected during the military conflict in Afghanistan [4] and a similar number affected since the start of the Syrian civil war [5-9].

$\mathrm{CL}$ is the second most important vector-borne disease in the Kingdom of Saudi Arabia (KSA) after dengue fever. Most of the reported CL cases are concentrated in the regions of $\mathrm{Al}$ Ahsa, $\mathrm{Al}$ Qassem, Riyadh, Asir, Hail and $\mathrm{Al}$ Madinah [10-22]. Since the early 1980', KSA has implemented a national CL control programme consisting not only in carrying out case detection in focus areas, but 
also involving vector and reservoir control. Due to this well-designed national control programme, the number of formally registered CL cases in KSA has dropped since 1987 , from $\sim 17,000$ to $\sim 2000$ cases per year in 2015 [14]. However, these official surveillance figures are likely to underestimate the prevalence and disease burden due to the number of unreported cases [14]. In addition to other non-communicable diseases, CL control in KSA is particularly important because of the millions of visitors that this country receives annually due to religious activities. Furthermore, around $35 \%$ of the country's work force consists of visitors arriving from other leishmaniasisendemic countries. Both anthroponotic (caused by $L$. tropica) $[10,17,22]$ and zoonotic (caused by $L$. major) $[6$, $15,23,24]$ CL have been reported in KSA, but very little is currently known about the national distribution of these parasite species.

Current drug treatment protocols for patients with Old World CL vary between EMR countries. Intralesional pentostam or sodium stibogluconate (SSG) is commonly used in this region, despite their high toxicity and the increasing number of unresponsive individuals. Currently, SSG is the second-line treatment choice for CL in KSA. In most cases, it is administered if the patient remains unresponsive (i.e. lack of re-epithelisation) after treatment with topical broad-spectrum antimicrobials (azoles and/or antibiotics; first line treatment). Topical paromomycin, either alone or in combination with gentamicin, was found to be effective in treatment of L. major cases in Tunisia [25], although it has not been implemented in KSA.

In this study, we present a complete epidemiological map of CL in KSA, including the clinical features associated with the two main parasite species, $L$. major and $L$. tropica. Furthermore, we show evidence that the efficacy of the current CL treatment protocol is highly dependent on the parasite species associated with the infection, geographical location and possibly also to the development of secondary infections in or around the lesion.

\section{Methods}

\section{Sample collection and clinical data}

Skin aspirate samples from a total of 104 adult CLpatients were collected from several cities or towns in KSA. Overall, this represented around $7 \%$ of all reported cases of CL in KSA in 2012. Case record studies and information sheets were obtained for all patients. This information was anonymised and re-labelled with the appropriate study code. The recorded information included all relevant clinical data (i.e. lesion size, number(s) and location(s) on the body, clinical features and treatment response, patient age and sex) and is provided in Additional file 1: Table S1.

\section{Parasite isolation and in vitro culture}

Parasite samples were collected by wound aspiration from Leishmania-infected patients after one month of lesion appearance using 200-300 $\mu \mathrm{l}$ of sterile PBS. Wound aspirates were transferred to plastic flasks (in triplicate) containing Leishmania culture medium M199 (Gibco, Grand Island, NY, USA) supplemented with $15 \%$ heat-inactivated foetal bovine serum (Invitrogen), 1.5\% BME vitamins (Sigma-Aldrich, Saint Louis, MO, USA) and $25 \mu \mathrm{g} / \mathrm{ml}$ gentamycin sulphate (SigmaAldrich, Saint Louis, MO, USA). Leishmania cultures were maintained for several days at $27^{\circ} \mathrm{C}$ and parasite DNA was extracted from logarithmic phase cultures using the DNeasy Blood and Tissue kit (Qiagen, Germantown, MD, USA) following the manufacturer's instructions.

\section{Drug treatment}

Clinically diagnosed patients were referred for treatment as recommended by the current KSA leishmaniasis treatment policy. This begins with the application of topical clotrimazole (1\%) and/or fusidic acid (2\%) for the treatment of secondary infections (i.e. bacterial, fungal or both). If after one week of treatment healing (re-epithelisation) was not initiated, the patient then received one course (of 14 injections each) of intralesional SSG $(20 \mathrm{mg} / \mathrm{kg} /$ day) (Additional file 2: Figure S1). All patients receiving SSG treatment were evaluated before and after treatment for complete blood counts and levels of aspartate aminotransferase, alanine aminotransferase, alkaline phosphatase, amylase and gamma glutamyltransferase. A few patients $(n=16)$ from the unsatisfactory responsive group (i.e. those with lesion extension, formation of satellite lesions or recurrence) were referred for a 3rd course of intramuscular SSG (20 mg/kg/day for up to 2 weeks) after clinical assessment (blood count, liver and renal function analyses).

\section{Identification of secondary infection species and antibiotic susceptibility testing}

Secondary infections were identified by clinical assessment. Nutrient agar, blood agar, MacConkey agar and Sabouraud dextrose agar were used for bacterial and fungal culture. Microscopy and biochemical assays were carried out to identify microbial species. Antibiotic susceptibility testing from identified species were performed using the semi-automatic analyser Microscan Autoscan-4 system (Beckman Coulter, Indianapolis, IN, USA). The antibiotics tested were ampicillin, azithromycin, cefoxitin, ciprofloxacin, erythromycin, fosfomycin, fusidic acid and gentamicin. All the laboratory analyses for microbial 
identification were carried out at King Abdulaziz Centre for Science and Technology (KACST).

\section{Species identification of Leishmania isolates}

Identification was performed based on a modified PCRrestriction fragment length polymorphism (PCR-RFLP) method previously described (26). A first PCR reaction of $25 \mu \mathrm{l}$ was set using the Q5 High-Fidelity 2X Mastermix (New England Biolabs, Ipswich, MA, USA) and the primers OL1853 and OL1854 at $200 \mathrm{nM}$. PCR conditions were as follows: $98^{\circ} \mathrm{C}$ for $1 \mathrm{~min}, 35$ cycles of $10 \mathrm{~s}$ at $98{ }^{\circ} \mathrm{C}, 30 \mathrm{~s}$ at $57{ }^{\circ} \mathrm{C}$ and $20 \mathrm{~s}$ at $72{ }^{\circ} \mathrm{C}$, and a final step at $72{ }^{\circ} \mathrm{C}$ for $1 \mathrm{~min}$. To increase the sensitivity, a second identical PCR was performed using $1 \mu \mathrm{l}$ of PCR product as template. Then, $20 \mu \mathrm{l}$ of the PCR product was incubated with HaeIII (New England Biolabs) in a $50 \mu \mathrm{l}$ reaction at $37{ }^{\circ} \mathrm{C}$ for 1 hour and inactivated at $80{ }^{\circ} \mathrm{C}$ for $20 \mathrm{~min}$. For the analysis, $5 \mu \mathrm{l}$ of unrestricted PCR product and $20 \mu \mathrm{l}$ of restricted product were run in a $2.5 \%$ agarose gel at 100 V. Leishmania species for each patient sample was determined by comparing its restriction pattern with $L$. major and L. tropica reference reactions. Unrestricted PCR products were also sequenced (Sanger sequencing; Source Biosciences, UK) to confirm product specificity.

\section{Data analysis}

All factors affecting the treatment responses were included in our analyses (i.e. clinical features, number and sites of the lesions, geographical area and parasite species). Fisher's exact test and Chi-square test were used to validate the statistical significance among the different factors. IMB SPSS Statistics 21 (IBM Corp. Released
2012. IBM SPSS Statistics for Windows, Version 21.0. Armonk, NY: IBM Corp.) was employed for all data analysis. Software ArcGIS 10 (ESRI 2011, ArcGIS Desktop: Release 10. Redlands, CA: Environmental Systems Research Institute) was used to map the distribution of parasite species and patient response to anti-leishmanial drug treatment across the regions, and in relation to elevation based on gridded Global Relief Data (ETOPO2) data, based on the geographical coordinates (latitude and longitude) of each site.

\section{Results \\ Identification of Leishmania species}

In KSA, Old World CL is mainly diagnosed by clinical assessment and microscopic examination, but not molecularly [10]. The infective Leishmania species is inferred based on the appearance, number of lesions and potential geographical location where infection may have occurred. Using a well-established PCR-RFLP analysis of the ITS1 region $[10,26,27]$ we identified the Leishmania species present in $104 \mathrm{CL}$ patients (Additional file 2: Figures S2-S5). All 104 CL patients were confirmed to be infected with either L. major or L. tropica. Leishmania major was the main species responsible for $C L$ in KSA and predominately found in the regions of $\mathrm{Al}$ Ahsa (East), Al Qassem and Riyadh (Central), and Al Madinah (Northwest) (Fig. 1 and Additional file 2: Figures S6-S7). Furthermore, L. tropica was the only species found in the Southwest regions of Asir and Jazan (Fig. 1 and Additional file 2: Figure S8). Interestingly, L. tropica was also detected in a few cases from the Al Madinah region, with one particular village reporting the presence of both $L$.

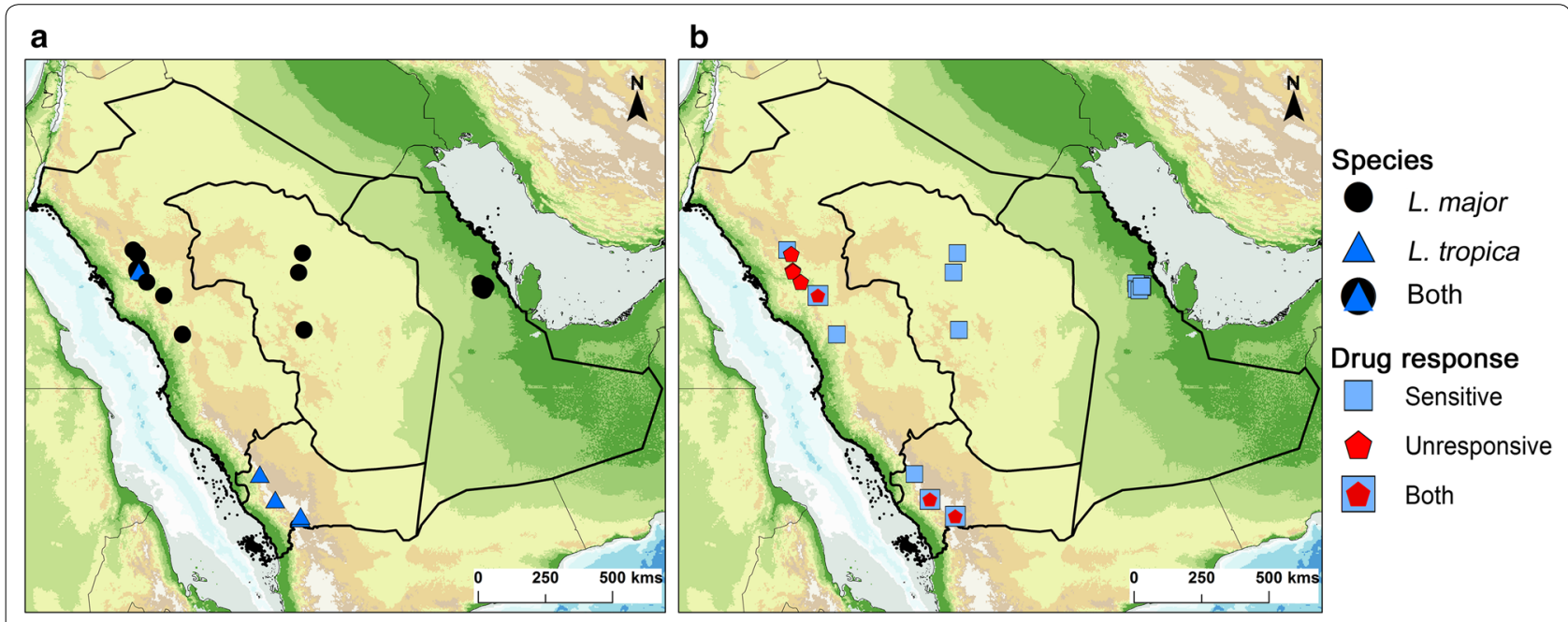

Fig. 1 Distribution of Leishmania species (a) and patient response to anti-leishmanial treatment (b) within the main CL endemic regions of Saudi Arabia. Map was created using software ArCGIS 10 (ESRI 2011, ArcGIS Desktop: Release 10. Redlands, CA: Environmental Systems Research Institute). See also detailed maps in Additional file 2: Figures S6, S7 and S8 
major and L. tropica infections (Fig. 1 and Additional file 2: Figure S3).

\section{Clinical presentations of CL patients vary depending on parasite species}

Several clinical features of the CL lesions, along with the infecting parasite species, were measured. These parameters included size, clinical presentations (i.e. papular, nodular or ulcerated nodular), location on the body, numbers, and whether satellite lesions were present (Table 1). Leishmania major lesions tended to be smaller $(16.5 \mathrm{~mm})$ than $L$. tropica ones $(22.2 \mathrm{~mm})$. The clinical presentations were significantly associated with the infecting species (Fisher's exact test of association; $P=0.001745$ ), with relatively similar numbers of each lesion type in $L$. major infections, but a notable skew towards ulcerated nodular lesions in L. tropica infections.

The location of lesions was also significantly associated with the infecting parasite species $(P \leq 0.00001)$, with $L$. tropica lesions appearing to be more common on the arms, face, nose or ear than L. major lesions, which are more common on other sites of the body (Table 1 ). Furthermore, the number of lesions was significantly associated with the parasite species $\left(P=7.201 \times 10^{-5}\right)$,

Table 1 Clinical presentation of $\mathrm{CL}$ lesions according to infecting parasite species

\begin{tabular}{|c|c|c|c|c|}
\hline Parasite species & & L. major & L. tropica & $P$-value \\
\hline Mean lesion size & & 16.5 & 22.2 & \\
\hline Lesion feature & Papular ${ }^{b}$ & 23 & 0 & 0.001745 \\
\hline & Nodular & 27 & 7 & \\
\hline & Ulcerated nodular ${ }^{c}$ & 25 & 14 & \\
\hline Lesion location ${ }^{d}$ & Hand, neck, head & 30 & 2 & $<0.00001$ \\
\hline & Arm & 7 & 12 & \\
\hline & Trunk, leg, foot & 19 & 0 & \\
\hline & Face, nose, ear & 3 & 7 & \\
\hline & Whole body & 16 & 0 & \\
\hline Lesion number & 1 & 23 & 10 & $<0.00001$ \\
\hline & 2 & 9 & 3 & \\
\hline & 3 & 5 & 8 & \\
\hline & 4 & 4 & 0 & \\
\hline & 5 & 7 & 0 & \\
\hline & $6+(\max .29)$ & 27 & 0 & \\
\hline Satellite lesions & Yes & 17 & 12 & 0.005813 \\
\hline & No & 58 & 9 & \\
\hline
\end{tabular}

\footnotetext{
${ }^{a}$ Fisher's exact test of association

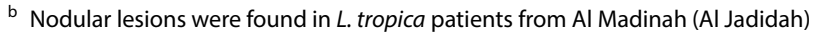
and Asir (Etoed)

c Ulcerated nodular lesions were found in two thirds of $C L$ patients. Lesions were mainly localized on the face, nose and/or ear

${ }^{d}$ The criteria used by the Saudi $\mathrm{MoH}$ for categorizing the location of the lesions is related to the use of traditional outfits, which determines the area of the body exposed to sand fly bites
}

with $L$. major infections displaying a bi-modal distribution of 1 lesion or $>6$ lesions while L. tropica infections all showed $\leq 3$ lesions. The presence of satellite lesions was also significantly associated with infecting species $(P=0.005813)$, with $57 \%$ of $L$. tropica infections displaying satellite lesions compared to $22 \%$ in L. major ulcers.

\section{Efficacy of anti-leishmanial treatment varies depending on parasite species and geographical location}

Confirmed (medically diagnosed) CL patients were referred for anti-leishmanial treatment starting with topical antifungals alone or in combination with antibiotics (first line of treatment) followed by 1-2 courses of IL SSG (as described in the Methods section). Overall, 30\% of $L$. major-infected patients responded to the first line of treatment alone and $82 \%$ after SSG courses were completed (Fig. 2a). However, none of the L. tropica-infected patients responded to topical azoles/fusidic acid, and in fact $60 \%$ did not respond at all to any treatment regimen even after receiving 2 courses of SSG (Table 2). Most $L$. tropica unresponsive patients withdrew after receiving the second course of SSG. Interestingly, when the treatment responses of patients from different geographical locations were compared, $90 \%$ of the cases from Central (i.e. Riyadh; exclusively L. major-infected) responded favourably (within 1 week) to topical azoles/fusidic acid alone (Fisher's exact test, $P=0.001$ ), whereas two-thirds of the cases from the Northwest (Al Madinah; mainly $L$. major-infected) and Southwest (Asir; exclusively L. tropica-infected) responded unsatisfactorily to both lines of treatment.

SIs were primarily detected in patients from the Eastern (35\%; exclusively L. major) and Southwest (40\%; exclusively $L$. tropica) regions (Fig. 2). Interestingly, $52 \%$ of $L$. major patients presenting SIs responded favourably after treatment with just topical azoles/fusidic acid (Fisher's exact test, $P=0.002$; Fig. $2 \mathrm{~b}$ ), with the remaining needing just one course of SSG to heal. Moreover, the group of $L$. major patients lacking SI responded poorly to azoles/fusidic acid treatment and many $(\sim 38 \%)$ remained unresponsive after a second SSG course. In contrast, the development of SIs did not have an effect upon the treatment response of $L$. tropica patients (Fig. 2b).

Staphylococcus species isolated from L. major lesions show resistance to a variety of antibiotics, including Fusidic Acid Staphylococcus epidermidis, S. haemolyticus and S. homins were isolated from $6 \mathrm{~L}$. major patients from $\mathrm{Al}$ Ahsa region. The resistance profiles (Fig. 3) showed that $6 / 6$ of $S$. epidermidis, 4/6 S. homins and 3/6 S. haemolyticus isolates are resistant to fusidic acid, which is part of the first line of CL treatment in KSA. From these 6 patients, 4 responded to the first line of treatment (azoles/fusidic 

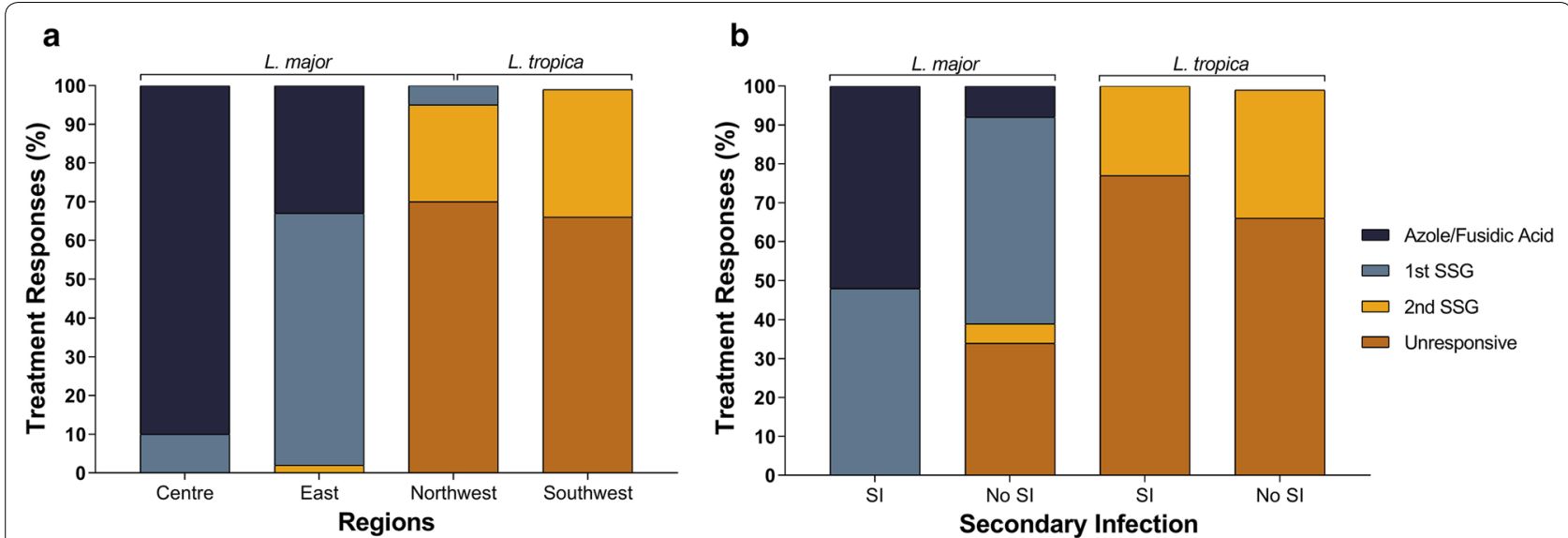

Fig. 2 Correlation between responses to drug treatment and CL endemic regions (a), and development of secondary infections (SI) and treatment response (b). a Distribution by region of 104 patients with L. major or L. tropica infections confirmed by culture, PCR-RFLP or clinical features. Patients from the Central region (exclusively infected with L. major) had a significantly higher response (healing; $P=0.001$, Fisher's exact test) to the first line of treatment compared to other $\mathrm{CL}$-endemic regions studied. In addition, patients from the Eastern region, responded significantly favorably ( $P=0.001$; Fisher's exact test) to the first course of SSG compared to those from both Northwest and Southwest regions. $\mathbf{b}$ Data for 96 patients with L. major $(n=75)$ or L. tropica $(n=21)$ infections confirmed by culture or PCR-RFLP. A significant $P=0.0002$ (Chi-square test) response to first line of treatment was observed only in L. major patients that had developed SI. However, there were no significant differences in L. tropica patients with or without SI. Abbreviations: SI, presence of secondary infections; No SI, absence of secondary infections

Table 2 Correlation between location of CL lesions and patient response to drug treatment. Data for 96 patients with $L$. major or $L$. tropica infections confirmed by culture or PCR-RFLP

\begin{tabular}{|c|c|c|c|c|c|}
\hline \multirow[t]{2}{*}{ Lesion location } & \multicolumn{5}{|c|}{ \% Patients responding to drug treatment (healing) } \\
\hline & $\begin{array}{l}\text { 1st line (Azoles/ } \\
\text { fusidic acid) }\end{array}$ & $\begin{array}{l}\text { 2nd line (SSG) 1st } \\
\text { course }\end{array}$ & $\begin{array}{l}\text { 2nd line (SSG) 2nd } \\
\text { course }\end{array}$ & 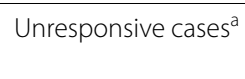 & P-value ${ }^{b}$ \\
\hline Head, neck or hand & $41(n=13)$ & $37(n=12)$ & $5(n=2)$ & $16(n=15)$ & $0.0192(n=32)$ \\
\hline Arms & $32(n=6)$ & $0(n=0)$ & $21(n=4)$ & $47(n=9)$ & $0.2(n=19)$ \\
\hline Trunk, legs or feet & $32(n=6)$ & $63(n=12)$ & $0(n=0)$ & $5^{c}(n=1)$ & $0.02(n=19)$ \\
\hline Face, nose or ear & $10(n=1)$ & $20^{d}(n=2)$ & $20(n=2)$ & $50^{e}(n=5)$ & $0.5(n=10)$ \\
\hline Disseminated (whole body) & $13(n=2)$ & $31(n=5)$ & $13(n=2)$ & $43^{f}(n=7)$ & $0.7(n=16)$ \\
\hline
\end{tabular}

a $85 \%$ of patients that did not respond to drug treatment were infected with L. tropica

b Fisher's exact test

c Ulcerated L. tropica cases with secondary infection

d All cases were L. major patients with apparent secondary infections

e Cases with satellite lesions

${ }^{f}$ Cases with multiple lesions receiving more than two courses of stibogluconate (SSG)

acid) and 2 had to receive 1 course of SSG to complete healing. Furthermore, all the Staphylococcus species isolated from $L$. major-infected patients showed resistance to azithromycin, whereas $S$. homins strains were resistant to ampicillin, cefoxitin and fosfomycin, and S. haemolyticus strains resistant to ampicillin and erythromycin.

\section{Discussion}

Several studies have investigated the clinical profiles of CL in Saudi Arabia [15-17, 20, 21, 28]. However, to our knowledge, this study is the most comprehensive dataset of CL samples collected from patients across the country. One hundred and four parasite isolates were collected from infected CL patients from the main endemic regions of KSA and the species molecularly identified by PCR-RFLP. Each patient was medically assessed and referred for treatment according to the KSA national treatment policy.

Leishmania major was found in patients from $\mathrm{Al}$ Ahsa, Riyadh, Al-Qassim and Al-Madinah, all from arid or semi-arid areas at a low altitude (not sea level), while $L$. tropica was exclusively detected in patients from Asir, 


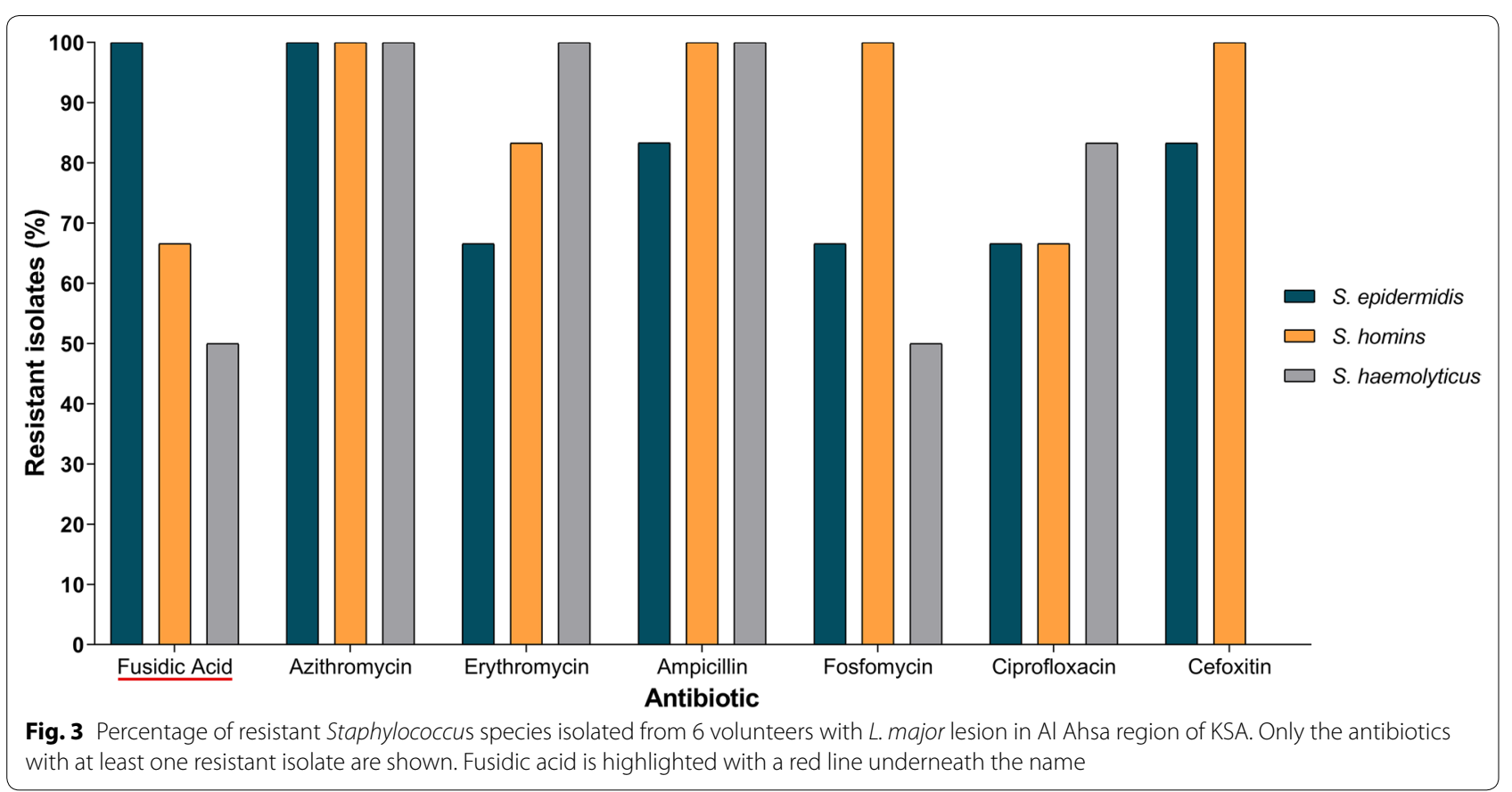

Jazan and Al-Jadidah (Al-Madinah) (high altitude) [29]. Interestingly, both $L$. major and L. tropica parasites were isolated in the Al-Jadidah village and from regions at similar altitude ( $\sim 600 \mathrm{~m}$ above sea level). We speculate that this is likely due to $L$. tropica-infected patients migrating to this region, as the climatic conditions and ecology of Al-Jadidah do not favour transmission of L. tropica by its main vector, Phlebotomus sergenti [29]. Furthermore, we have recently characterized the sand fly vectors from the same Al-Madinah locations and found no evidence of $P h$. sergenti in this region, although it has been previously reported in other areas [11, 15, 22, 29].

The clinical features of $L$. major patients appear to vary according to the endemic area [28]. Although we did not find statistically significant differences between the different regions sampled in this study, we observed that ulcerated nodular lesions were found predominantly in cases from $\mathrm{Al}$ Ahsa, whereas nodular and papular lesions were common in Al Madinah and Riyadh. Furthermore, multiple lesions were found in over half of L. major patients from $\mathrm{Al}$ Ahsa. This differs from previous reports where only $5 \%$ of the patients (presumably also infected with $L$. major) presented multiple lesions [28, 30]. These differences may be due in part to higher exposure of people to sand fly bites as most of the CL patients from $\mathrm{Al}$ Ahsa that took part in this study were migrant construction workers.

Several factors may account for differences in treatment response, including the possible presence of different drug-resistant parasites, the nutritional and immune status of the patients and their genetic backgrounds. In addition, sand fly saliva could also play an important role in disease outcome as saliva from Ph. papatasi (L. major vector) has been reported to trigger either a Type-I (regarded as protective against a Leishmania infection) or II delayed-type hypersensitivity in healthy individuals from CL endemic areas [31]. Furthermore, we recently showed that previous exposure to $P h$. papatasi bites appears to influence the severity of CL [29]. As for Ph. sergenti saliva [32], nothing is known about its effect in the human immune system or in modulating CL pathology. The presence of ulcerated nodular lesions also showed a strong correlation with L. tropica infection in patients from all the $\mathrm{CL}$ endemic regions where this species was found. Therefore, the infection with each of the parasite species appears to favour the development of specific clinical feature(s), which vary depending on geography and may also impact treatment response. More research is needed to establish a correlation between treatment efficacy and the different clinical presentations of Old World CL patients.

Approximately $50 \%$ of the L. major patients (mainly from the Central or Eastern regions) who presented with ulcerated nodular lesions had detectable SI. Interestingly, there was a statistically significant (Fisher's exact test, $P=0.0001$ ) correlation with the response to re-epithelisation after treatment with azole/fusidic acid (without further administration of SSG) in patients from the Central region compared to patients from other endemic areas (Fig. 2). This suggests that elimination of the SI appears 
to favour healing of L. major patients from Riyadh and Al Ahsa, but has little effect in those from Al Madinah, although the lack of response of the latter may be also correlate with the time of residency in this region [27, 28]. In addition, we cannot rule out that first line of treatment could accelerate re-epithelization in patients who may have self-healed over time. However, this seems unlikely because most of the patients seek medical assistance after the lesion has worsened. Moreover, like pathogenic fungi, Leishmania parasites make ergosterol, an essential membrane lipid, the synthesis of which is inhibited by azoles [33]. Thus, treatment with topical azoles alone may possibly impact directly on $L$. major susceptible strains. Also, elimination of the fungal or bacterial infection may boost the immune system to fight the L. major infection in many cases, with the exception being those presenting papular lesions from the $\mathrm{Al}$ Madinah region. Alternatively, the indirect effect that the development of SIs may have in the treatment of $L$. major-infected patients could also be a result of the release of bacterial or fungal molecules after application of the first line of treatment. This could activate host immunity and contribute to the elimination of $L$. major infection and subsequent activation of wound healing mechanisms [34]. Currently, we are performing an extensive metagenomic analysis to characterise the microbiota present in lesions from both L. major and L. tropica patients. These results will be key to associate specific microbial groups with differences in treatment response.

It is worth mentioning that in some cases the first line of treatment is applied to increase efficacy of SSG regime. Regarding L. tropica-infected patients, most were unresponsive (60\%) to the first course of anti-leishmanial treatment and healing only occurred in patients receiving a second (and sometimes a third) course of SSG. Unresponsive cases to SSG have been reported among L. tropica patients in neighbour countries like Iran [35]. Moreover, it remains to be determined whether L. tropica strains from KSA are less sensitive to azoles or alternatively, the drug may be more accessible to the parasite in L. major lesions than in L. tropica ones.

Novel therapies against CL need to be evaluated in clinical trials in KSA. For example, the oral administration of miltefosine, an alkylphosphocholine analogue that inhibits phospholipid and sterol biosynthesis, proved to be safer and more effective than SSG for the treatment of CL caused by L. braziliensis in Brazil [36]. Likewise, it would be interesting to test the efficacy of topical paromomycin for the treatment of L. tropica infections [25]. None of these drugs is used in the current CL treatment regime in KSA but could represent alternative approaches in unresponsive cases.
Taken together, differences in the susceptibility to either azoles or SSG among parasite strains and resistance against fusidic acid observed for the Staphylococcus species isolated from $L$. major CL lesions suggest that the current protocols for CL treatment need to consider not only the parasite species, but also the geographical endemicity of CL infections. Further research is required to understand whether treatment unresponsiveness in KSA is also due to the development of drug-resistant parasites. Knowing this will help determine if different antileishmanial treatment protocols need to be considered.

\section{Conclusions}

The findings of this study demonstrate that patient responses to current anti-leishmanial treatment vary between the different CL endemic areas and are also partially dependent on the development of SIs. These results have implications for the implementation of differential treatment regimens according to the CL-endemic area, which in turn will save financial resources and ensure patients are treated with the most efficacious antileishmanial therapies. It remains to be determined in CL-endemic areas from other EMR countries whether such profound differences in anti-Leishmania treatment responses are also observed among CL patients from different geographical locations.

\section{Additional files}

Additional file 1: Table S1. Cohort characteristics and relevant clinical features.

Additional file 2: Figure S1. Scheme representing the current leishmaniasis treatment policy in KSA. Figure S2. Leishmania spp. identification in Central Region by PCR-RFLP analysis of parasite ITS1 region. Lane Lt: L. tropica positive control; Lane Lm: L. major positive control; Lanes 1-10: different examples of Leishmania isolates from Rass, Dwadmi and Muzahmyyah. Figure S3. Leishmania spp identification in Al Madinah Province by PCR-RFLP analysis of parasite ITS1 region. Lanes 1-4: different examples of Leishmania isolates from Aljadaida and Sulailah, Al Madinah Province; Lanes 1 and 2: L. tropica samples; Lanes 3 and 4: L. major samples; Lane Lm: L. major positive control; Lane Lt: L. tropica positive control. Figure S4. Leishmania spp. identification in Al Ahsa Region by PCR-RFLP analysis of parasite ITS1 region. Lanes 1-13: different examples of Leishmania isolates from Al Ahsa Region; Lane Lt: L. tropica positive control; Lane Lm: L. major positive control. Figure S5. Leishmania spp identification in Asir Region by PCR-RFLP analysis of parasite ITS1 region. Lanes 1-5: different examples of Leishmania isolates from Asir Province. Figure S6. Distribution of Leishmania species (a) and patient response to anti-leishmanial treatment (b) within the Eastern region of Saudi Arabia. The map was created using software ArcGIS 10 (ESRI, Redlands, CA). Figure S7. Distribution of Leishmania species (a) and patient response to anti-leishmanial treatment (b) within the Northwest region of Saudi Arabia. The map was created using software ArcGIS 10 (ESRI, Redlands, CA). Figure S8. Distribution of Leishmania species (a) and patient response to anti-leishmanial treatment (b) within the southwest region of Saudi Arabia. The map was created using software ArcGIS 10 (ESRI, Redlands, CA). 


\begin{abstract}
Abbreviations
KSA: Kingdom of Saudi Arabia; CL: cutaneous leishmaniasis; SSG: sodium stibogluconate; SI: secondary infections; EMR: East Mediterranean Region; $\mathrm{MoH}$ : Ministry of Health.
\end{abstract}

\section{Acknowledgements}

We are indebted to colleagues from the Saudi MoH who helped in all aspects related to sample collection, especially to Dr Mohammed Adnan Aldahan and Mr Khalid Alsuhaibani (Riyadh), Mr Ahmed Yassin Almuhanaa (Al Ahsa) Mr Salim Alzubiani and Mr Alkhir Dafeallah (Al Madinah), and Mr Abdulaziz AL Jarallah (Asir). We thank Dr Turki Saud Mohammed Al-Saud (KACST president) for his kindness in allowing access to KACST culture facilities, Dr Lee Haines, Dr Deirdre Walshe and Dr Krishanthi Subramaniam for manuscript editing, Dr lan Hasting for valuable statistical advice, and Mrs Rabab Al-Malki for artistic work.

\section{Funding}

This work was partially funded by a PhD studentship from the Saudi Cultural Bureau (awarded to WSAS); GlycoPar-EU FP7 Marie Curie Initial Training Network (GA. 608295 awarded to ACS and AAS; http://www.ec.europa.eu); Shefa Fund (awarded to AAS). The funders had no role in study design, data collection and analysis, decision to publish, or preparation of the manuscript.

\section{Availability of data and materials}

All data generated or analysed during this study are included in this published article.

\section{Authors' contributions}

WSA contributed to conceiving, designing, conducting and analysing the experiments, design of the study and manuscript writing. CS contributed to conducting, analysing the experiments and manuscript writing. GDW, NAD, $\mathrm{LKH}, \mathrm{ACS}, \mathrm{YA}, \mathrm{EJA}$, AH contributed to conducting and analysing experiments. $\mathrm{SMB}, \mathrm{KSA}, \mathrm{ZA}$, AMAS contributed to sample collection. MHA, AMA, ZM contributed in study design. AAS contributed to conceiving, designing and analysing experiments, design of the study and manuscript writing, with input from all authors. All authors read and approved the final manuscript.

\section{Ethics approval and consent to participate}

Ethical approval was obtained from both from the Liverpool School of Tropical Medicine, UK (12.03RS) and the Saudi Ministry of Health $(\mathrm{MoH})$ Ethical Committees. All patients provided written informed consent for the collection of biological samples and subsequent analyses. All patient data were anonymized. Furthermore, all research was conducted according to Declaration of Helsinki principles.

\section{Consent for publication}

Not applicable.

\section{Competing interests}

The authors declare that they have no competing interests.

\section{Publisher's Note}

Springer Nature remains neutral with regard to jurisdictional claims in published maps and institutional affiliations.

\begin{abstract}
Author details
1 Department of Tropical Disease Biology, Liverpool School of Tropical Medicine, Liverpool, UK. ${ }^{2}$ Present Address: National Centre for Tropical Diseases, Saudi Ministry of Health, Riyadh, Kingdom of Saudi Arabia. ${ }^{3}$ Department of Clinical Sciences, Liverpool School of Tropical Medicine, Liverpool, UK. ${ }^{4}$ Faculty of Sciences, Liverpool John Moores University, Liverpool, UK. ${ }^{5}$ National Center for Biotechnology, King Abdulaziz City for Science and Technology, Riyadh, Saudi Arabia. ${ }^{6}$ School of Cellular and Molecular Medicine, University of Bristol, Bristol, UK. ${ }^{7}$ Saudi Ministry of Health, Riyadh, Kingdom of Saudi Arabia. ${ }^{8}$ Antimicrobial Research Centre, University of Leeds, Leeds, UK. ${ }^{9}$ Department of Vector Biology, Liverpool School of Tropical Medicine, Liverpool, UK.
\end{abstract}

Received: 21 December 2018 Accepted: 20 April 2019

Published online: 02 May 2019

\section{References}

1. Postigo JAR. Leishmaniasis in the World Health Organization Eastern Mediterranean Region. Int J Antimicrob Agents. 2010;36:S62-5.

2. Alvar J, Vélez ID, Bern C, Herrero M, Desjeux P, Cano J, et al. Leishmaniasis worldwide and global estimates of its incidence. PLoS One. 2012;7:e35671.

3. World Health Organization. Control of the leishmaniases. World Health Organ Tech Rep Ser. 2010;7-8:1-186.

4. Wallace MR, Hale BR, Utz GC, Olson PE, Earhart KC, Thornton SA, et al. Endemic infectious diseases of Afghanistan. Clin Infect Dis. 2002;34:S171-207.

5. Alasaad S. War diseases revealed by the social media: massive leishmaniasis outbreak in the Syrian Spring. Parasit Vectors. 2013;6:94.

6. Salam N, Al-Shaqha WM, Azzi A. Leishmaniasis in the Middle East: incidence and epidemiology. PLoS Negl Trop Dis. 2014;8:e3208.

7. Saroufim M, Charafeddine K, Issa G, Khalifeh H, Habib RH, Berry A, et al. Ongoing epidemic of cutaneous leishmaniasis among Syrian refugees, Lebanon. Emerg Infect Dis. 2014;20:1712-5.

8. Sharara SL, Kanj SS. War and infectious diseases: challenges of the Syrian civil war. PLoS Pathog. 2014;10:e1004438.

9. Al-Salem WS, Pigott DM, Subramaniam K, Haines LR, Kelly-Hope L, Molyneux DH, et al. Cutaneous leishmaniasis and conflict in Syria. Emerg Infect Dis. 2016;22:931-3.

10. Al-Salem WS, Ferreira FM, Dyer NA, Alyamani EJ, Balghonaim SM, AIMehna AY, et al. Detection of high levels of anti-a-galactosyl antibodies in sera of patients with Old World cutaneous leishmaniasis: a possible tool for diagnosis and biomarker for cure in an elimination setting. Parasitology. 2014;141:1898-903.

11. El-Badry A, Al-Juhani A, Ibrahim E-K, Al-Zubiany S. Distribution of sand flies in El-Nekheil province, in Al-Madinah Al-Munawwarah region, western of Saudi Arabia. Parasitol Res. 2008;103:151-6.

12. Al-Zahrani MA, Peters W, Evans DA, Chin C, Smith V, Lane RP. Phlebotomus sergenti, a vector of Leishmania tropica in Saudi Arabia. Trans R Soc Trop Med Hyg. 1988;82:416.

13. Ibrahim EA, Al-Zahrani MA, Al-Tuwaigri AS, Al-Shammary FJ, Evans DA. Leishmania infecting man and wild animals in Saudi Arabia. 9. The black rat (Rattus rattus) a probable reservoir of visceral leishmaniasis in Gizan Province, south-west Saudi Arabia. Trans R Soc Trop Med Hyg. 2017;86:513-4.

14. Abuzaid AA, Abdoon AM, Aldahan MA, Alzahrani AG, Alhakeem RF, Asiri AM, et al. Cutaneous leishmaniasis in Saudi Arabia: a comprehensive overview. Vector Borne Zoonotic Dis. 2017:17:673-84.

15. El-Beshbishy HA, Al-Ali KH, El-Badry AA. Molecular characterization of cutaneous leishmaniasis in Al-Madinah Al-Munawarah Province, western Saudi Arabia. Int J Infect Dis. 2013;17:e334-8.

16. Dye C, Killick-Kendrick R, Ismail RB, Al-Gindan Y. Zoonotic cutaneous leishmaniasis in Saudi Arabia: results of a preliminary epidemiological survey in Al-Ahsa oasis. Trans R Soc Trop Med Hyg. 1989;83:493-8.

17. Al-Zahrani MA, Peters W, Evans DA, Smith V, Ching Chin I. Leishmania infecting man and wild animals in Saudi Arabia. 6. Cutaneous leishmaniasis of man in the south-west. Trans R Soc Trop Med Hyg. 1989;83:621-8.

18. Ibrahim EA, Mustafa MB, Al Amri SA, Al-Seghayer SM, Hussein SM, Gradoni L. Meriones libycus (Rodentia: Gerbillidae), a possible reservoir host of zoonotic cutaneous leishmaniasis in Riyadh Province, Saudi Arabia. Trans R Soc Trop Med Hyg. 1994;88:39.

19. El Sibae MM, Eesa NM, Morsy TA. Rodents and cutaneous leishmaniasis in Qasim, Saudi Arabia. J Egypt Soc Parasitol. 1993;23:667-73.

20. Khan W, Zakai HA. Epidemiology, pathology and treatment of cutaneous leishmaniasis in Taif region of Saudi Arabia. Iran J Parasitol. 2014;9:365-73.

21. El-Sibae MM, Eesa NM. A study on Phlebotomus species, the vectors of leishmaniasis in Gassim, Saudi Arabia. J Egypt Soc Parasitol. 1993;23:231-8.

22. El-Beshbishy HA, Al-Ali KH, El-Badry AA. Molecular characterization of Leishmania infection in sand flies from Al-Madinah Al-Munawarah Province, western Saudi Arabia. Exp Parasitol. 2013;134:211-5.

23. Al-Gindan $Y$, Omer AHS, A-Humaidan Y, Peters W, Evans DA. A case of mucocutaneous leishmaniasis in Saudi Arabia caused by Leishmania major and its response to treatment. Clin Exp Dermatol. 1983;8:185-8. 
24. Elbihari S, Kawasmeh ZA, Al-Naiem A, Al-Atiya S. Leishmania infecting man and wild animals in Saudi Arabia. 3. Leishmaniasis in Psammomys obesus Cretzschmar in Al-Ahsa oasis. Trop Med Parasitol. 1987;38:89-92.

25. Ben Salah A, Ben Messaoud N, Guedri E, Zaatour A, Ben Alaya N, Bettaieb J, et al. Topical paromomycin with or without gentamicin for cutaneous leishmaniasis. N Engl J Med. 2013;368:524-32.

26. Schönian G, Nasereddin A, Dinse N, Schweynoch C, Schallig HDF, Presber W, et al. PCR diagnosis and characterization of Leishmania in local and imported clinical samples. Diagn Microbiol Infect Dis. 2003:47:349-58.

27. El Tai NO, Osman OF, El Fari M, Presber W, Schönian G. Genetic heterogeneity of ribosomal internal transcribed spacer in clinical samples of Leishmania donovani spotted on filter paper as revealed by single-strand conformation polymorphisms and sequencing. Trans R Soc Trop Med Hyg. 2000;94:575-9.

28. Al-Tawfiq JA, AbuKhamsin A. Cutaneous leishmaniasis: a 46-year study of the epidemiology and clinical features in Saudi Arabia (1956-2002). Int J Infect Dis. 2004;8:244-50.

29. Mondragon-Shem K, Al-Salem WS, Kelly-Hope L, Abdeladhim M, Al-Zahrani MH, Valenzuela JG, et al. Severity of Old World cutaneous leishmaniasis is influenced by previous exposure to sandfly bites in Saudi Arabia. PLoS Negl Trop Dis. 2015;9:e0003449.

30. Al-Qurashi AR, Ghandour AM, Osman M, Al-Juma M. Dissemination in cutaneous leishmaniasis due to Leishmania major in different ethnic groups in Saudi Arabia. Int J Dermatol. 2000;39:832-6.
31. Oliveira F, Traoré B, Gomes R, Faye O, Gilmore DC, Keita S, et al. Delayedtype hypersensitivity to sand fly saliva in humans from a leishmaniasisendemic area of Mali is TH1-mediated and persists to midlife. J Invest Dermatol. 2013;133:452-9.

32. Rohoušová I, Subrahmanyam S, Volfová V, Mu J, Volf P, Valenzuela JG, et al. Salivary gland transcriptomes and proteomes of Phlebotomus tobbi and Phlebotomus sergenti, vectors of leishmaniasis. PLoS Negl Trop Dis. 2012;6:e1660

33. Vanden Bossche H, Marichal P, Gorrens J, Coene MC, Willemsens G, Bellens D, et al. Biochemical approaches to selective antifungal activity. Focus on azole antifungals. Mycoses. 1989;32(Suppl. 1):35-52.

34. Lopes MEM, Carneiro MBH, dos Santos LM, Vieira LQ. Indigenous microbiota and leishmaniasis. Parasite Immunol. 2016;38:37-44.

35. Hadighi R, Mohebali M, Boucher P, Hajjaran H, Khamesipour A, Ouellette M. Unresponsiveness to glucantime treatment in Iranian cutaneous leishmaniasis due to drug-resistant Leishmania tropica parasites. PLoS Med. 2006;3:e162.

36. Machado PR, Ampuero J, Guimarães LH, Villasboas L, Rocha AT, Schriefer A, et al. Miltefosine in the treatment of cutaneous leishmaniasis caused by Leishmania braziliensis in Brazil: a randomized and controlled trial. PLoS Negl Trop Dis. 2010;4:e912.
Ready to submit your research? Choose BMC and benefit from:

- fast, convenient online submission

- thorough peer review by experienced researchers in your field

- rapid publication on acceptance

- support for research data, including large and complex data types

- gold Open Access which fosters wider collaboration and increased citations

- maximum visibility for your research: over 100M website views per year

At BMC, research is always in progress.

Learn more biomedcentral.com/submissions 